AS-ITP-2000-009

September, 2000

\title{
Superconformal Algebras in Light-cone Gauge Quantization of String Theories on $A d S_{3}$
}

\author{
JIAN JING' and MING YU P \\ Institute of Theoretical Physics, Academia Sinica \\ P.O.Box 2735, Beijing 100080, P.R.China
}

\begin{abstract}
Motivated by superstring theories on $A d S_{3}$, we construct spacetime superconformal algebras (SCAs) living on the $A d S_{3}$ boundary in terms of the transversal physical degrees of freedom. The SCAs constructed are $\mathrm{N}=4$ large, middle algebras, and $N=3$ algebra, corresponding to superstring theories on $A d S_{3} \times S^{3} \times S^{3} \times S^{1}, A d S_{3} \times S^{3} \times T^{4}$ and $A d S_{3} \times\left(S^{3} \times S^{3} \times S^{1}\right) / Z_{2}$ backgrounds respectively.
\end{abstract}

${ }^{1}$ e-mail: jingj@itp.ac.cn

2e-mail: yum@itp.ac.cn 


\section{Introduction}

String theory on $A d S$ space has been studied intensively, [1, 2, 3, 4] in particular, with the connection to Maldacena's conjecture [5, 11, 12, 13, 14, 6, 7, 8, 9, 10. A precise statement on the $A d S / C F T$ correspondence has been given in ref. [15, 16]. In ref. [17, 18, the spacetime CFT living on the boundary of $A d S_{3}$ were studied and the $N=4$ superconformal algebras on the spacetime boundary were constructed. Spacetime properties of superstring theory on $A d S_{3} \times S^{3} \times S^{3} \times S^{1}$ were studied in ref.[20], and the "large" $N=4$ algebras were constructed. Both of those algebras were constructed in the covariant form. In ref.[21] the light-cone gauge quantization of string theory on $A d S_{3}$ was given and shown to be equal to the covariant one, provided the center charge is 26 (Bosonic) or 15 (Fermionic). In the light-cone gauge, the spacetime superconformal algebra (SCA) were constructed in terms of the transversal physical degrees of freedom [21]. In this note we shall go along this line further. That is, we shall construct spacetime large, middle $N=4$ [22, 23] and $N=3$ [24] SCAs in terms of the transversal physical degrees of freedom, corresponding to superstring theories on $A d S_{3} \times S^{3} \times S^{3} \times S^{1}$, $A d S_{3} \times S^{3} \times T^{4}$ and $A d S_{3} \times\left(S^{3} \times S^{3} \times S^{1}\right) / Z_{2}$ spacetime background respectively.

This note is organized as follows. In section 2 , we introduce our notations first, then we construct the large $N=4 \mathrm{SCF}$ algebra. In section 3 , we get middle $N=4 \mathrm{SCF}$ algebra as well as $N=3 \mathrm{SCF}$ algebra from the large one by taking certain limits. Some remarks are given in the last section.

\section{$2 \quad$ Large $N=4$ spacetime SCFT}

In this section we construct large $N=4 \mathrm{SCF}$ algebra, which corresponds to string propagating on $A d S_{3} \times S^{3} \times S^{3} \times S^{1}$ background. In Neveu-Schwarz-Ramond formalism, the worldsheet supersymmetry introduces ten worldsheet Majorana fermions, which however, transform as spacetime vectors. There are three worldsheet fermions $\psi^{A}$, "A" being the vector indices on the $S L(2)$ manifold, and similarly three fermions $\chi^{a}$ from the first $S U(2)$ manifold, three fermions $\omega^{a}$ from the second $S U(2)$ manifold, and a single fermion $\lambda$ from the $U(1)$ part. Our convention are almost the same as in ref. [20] and [21]. The OPEs among them are:

$$
\begin{aligned}
\psi^{A}(z) \psi^{B}(w) & =\frac{\eta^{A B}}{z-w} \\
\chi^{a}(z) \chi^{b}(w) & =\omega^{a}(z) \omega^{b}(w)=\frac{\delta^{a b}}{z-w} \\
\lambda(z) \lambda(w) & =\frac{1}{z-w}
\end{aligned}
$$

where $\eta^{A B}=\operatorname{diag}(+,+,-)$.

In the $S L(2) \times S U(2) \times S U(2) \times U(1)$ super WZNW model, there are affine bosonic currents, $j^{A}, k^{i}, m^{i}$ with level $k+2, k^{\prime}-2, k^{\prime \prime}-2$, respectively. The OPEs among them are

$$
\begin{aligned}
j^{A}(z) j^{B}(w) & =\frac{\frac{k+2}{2} \eta^{A B}}{(z-w)^{2}}+\frac{i \eta_{C D} \epsilon^{A B C} j^{D}}{z-w} \\
k^{i}(z) k^{j}(w) & =\frac{\frac{k^{\prime}-2}{2} \delta^{i j}}{(z-w)^{2}}+\frac{i \epsilon^{i j k} k^{k}}{z-w}
\end{aligned}
$$




$$
\begin{aligned}
m^{i}(z) m^{j}(w) & =\frac{\frac{k^{\prime \prime}-2}{2} \delta^{i j}}{(z-w)^{2}}+\frac{i \epsilon^{i j k} m^{k}}{z-w} \\
\partial Y(z) \partial Y(w) & =-\frac{1}{(z-w)^{2}}
\end{aligned}
$$

The criticality of fermionic string, $c=15$, gives the following relation [20]

$$
\frac{1}{k}=\frac{1}{k^{\prime}}+\frac{1}{k^{\prime \prime}}
$$

The $\operatorname{sl}(2)$ current algebra can be constructed in terms of the three bosons as follows,

$$
\begin{aligned}
j^{3} & =\beta \gamma+\frac{\alpha_{+}}{2} \partial \phi \\
j^{+} & =\beta \gamma^{2}+\alpha_{+} \gamma \partial \phi+k \partial \gamma \\
j^{-} & =\beta
\end{aligned}
$$

where $\alpha_{+}=\sqrt{2 k}$. The OPEs of $\beta, \gamma, \phi$ are

$$
\begin{aligned}
\phi(z) \phi(w) & =-\log (z-w)+\cdots \cdots \\
\beta(z) \gamma(w) & =\frac{1}{z-w}+\cdots \cdots
\end{aligned}
$$

Here, $j^{+}=j^{1}+i j^{2}, j^{-}=j^{1}-i j^{2}$.

When the light-cone gauge [21] $\gamma=z^{p}$, and $\tilde{\psi}^{+}=0$ is imposed, only $\phi$ and $\tilde{\psi}^{3}$ (The OPE can be normalized as $\left.\tilde{\psi}^{3}(z) \tilde{\psi}^{3}(w)=\frac{1}{z-w}\right)$ from $A d S_{3}$ part are dynamical ones. The bosonic part of the spacetime Virasoro generators are

$$
L_{n}=\oint d z\left[-\frac{1}{2} \partial \phi \partial \phi-\left(\frac{1}{\alpha_{+}}-\frac{\alpha_{+}}{2}\right) \partial^{2} \phi+\frac{1}{k^{\prime}} \sum k^{i} k^{i}+\frac{1}{k^{\prime \prime}} \sum m^{i} m^{i}-\frac{1}{2} \partial Y \partial Y-\frac{\Delta}{z^{2}}\right] e^{n q} z^{n p+1}
$$

Now we decompose the transversal physical degrees of freedom into the representations of the $s u(2) \times s u(2)$. The Liouville field $\phi$ and its super partner $\left(\tilde{\psi}^{3}\right)$ from the $A d S_{3}$ are in the $(\mathbf{0}, \mathbf{0})$. The $k^{i}$ 's and their super partners $\chi^{a}$ 's are in the $(\mathbf{1}, \mathbf{0})$. Fields from $U(1)$ and its super partner are in the $(\mathbf{0}, \mathbf{0})$, and $m^{i}$ s and their super partners $\omega^{a}$ in the $(\mathbf{0}, \mathbf{1})$. Since the spacetime supercurrents $G_{\alpha \beta}$ 's are spacetime spinors, and the worldsheet fermions are spacetime vectors, we first need to transform the later into spacetime spinors. This can be done by the usual bosonization procedure, as in ref. [21]

Define

$$
\begin{aligned}
\chi^{3}+\tilde{\psi}^{3} & =e^{\phi^{1}} \\
\chi^{3}-\tilde{\psi}^{3} & =e^{-\phi^{1}} \\
\chi^{+} & =e^{\phi^{2}} \\
\chi^{-} & =e^{-\phi^{2}} \\
\lambda^{3}+i \lambda^{0} & =e^{\phi^{3}} \\
\lambda^{3}-i \lambda^{0} & =e^{-\phi^{3}} \\
\lambda^{1}+i \lambda^{2} & =e^{\phi^{4}} \\
\lambda^{1}-i \lambda^{2} & =e^{-\phi^{4}}
\end{aligned}
$$


Then we can construct the global $s u(2) \times s u(2)$ generators acting on these fermions in terms of the $\phi^{A}$ 's

$$
\begin{aligned}
j^{+} & =\oint e^{\phi^{2}}\left(e^{\phi^{1}}+e^{-\phi^{1}}\right) \\
j^{-} & =\oint\left(e^{\phi^{1}}+e^{-\phi^{1}}\right) e^{-\phi^{2}} \\
j^{3} & =\oint \partial \phi^{2} \\
k^{+} & =\oint e^{\phi^{4}}\left(e^{\phi^{3}}+e^{-\phi^{3}}\right) \\
k^{-} & =\oint\left(e^{\phi^{3}}+e^{-\phi^{3}}\right) e^{-\phi^{4}} \\
k^{3} & =\oint \partial \phi^{4}
\end{aligned}
$$

Using the $\phi^{A}$ fields and considering the representations of the $j^{a}$ 's and $k^{a}$ 's, we can construct a set of worldsheet fermions which are also spacetime spinors

$$
\begin{aligned}
\psi_{1} & =e^{\frac{1}{2}\left(\phi_{1}+\phi_{2}+\phi_{3}+\phi_{4}\right)} \\
\bar{\psi}_{1} & =e^{\frac{1}{2}\left(-\phi_{1}-\phi_{2}-\phi_{3}-\phi_{4}\right)} \\
\psi_{2} & =e^{\frac{1}{2}\left(-\phi_{1}-\phi_{2}+\phi_{3}+\phi_{4}\right)} \\
\bar{\psi}_{2} & =e^{\frac{1}{2}\left(\phi_{1}+\phi_{2}-\phi_{3}-\phi_{4}\right)} \\
\chi_{1} & =e^{\frac{1}{2}\left(-\phi_{1}+\phi_{2}+\phi_{3}-\phi_{4}\right)} \\
\bar{\chi}_{1} & =e^{\frac{1}{2}\left(\phi_{1}-\phi_{2}-\phi_{3}+\phi_{4}\right)} \\
\chi_{2} & =e^{\frac{1}{2}\left(\phi_{1}-\phi_{2}+\phi_{3}-\phi_{4}\right)} \\
\bar{\chi}_{2} & =e^{\frac{1}{2}\left(-\phi_{1}+\phi_{2}-\phi_{3}+\phi_{4}\right)}
\end{aligned}
$$

where $\phi_{i}$ satisfy $\phi_{i}(z) \phi_{j}(w)=\delta_{i j} \log (z-w)$, and the OPEs of $\psi_{\alpha}, \chi_{\alpha}$ are

$$
\begin{aligned}
\psi_{\alpha}(z) \bar{\psi}_{\beta}(w) & =\frac{\delta_{\alpha \beta}}{z-w}+\cdots \\
\chi_{\alpha}(z) \bar{\chi}_{\beta}(w) & =\frac{\delta_{\alpha \beta}}{z-w}+\cdots
\end{aligned}
$$

and all others are regular. It is easy to see that both $\psi_{i}$ 's and $\chi_{i}$ 's are doublets of $j^{i}$ and $k^{i}$, $j^{i}$ acts from the left, and $k^{i}$ from the right [21]. The contributions to the spacetime energymomentum tensor from fermions in terms of $\psi$ 's, $\bar{\psi}$ 's and $\chi$ 's, $\bar{\chi}$ 's can be expressed as [21]

$$
T_{F}=\frac{1}{2}\left(\partial \psi_{1} \bar{\psi}_{1}-\psi_{1} \partial \bar{\psi}_{1}+\partial \psi_{2} \bar{\psi}_{2}-\psi_{2} \partial \bar{\psi}_{2}+\partial \chi_{1} \bar{\chi}_{1}-\chi_{1} \partial \bar{\chi}_{1}+\partial \chi_{2} \bar{\chi}_{2}-\chi_{2} \partial \bar{\chi}_{2}\right)
$$

where we have set $q=0, p=1$, so we can regard

$$
T=-\frac{1}{2} \partial \phi \partial \phi-\left(\frac{1}{\alpha_{+}}-\frac{\alpha_{+}}{2}\right) \partial^{2} \phi+\frac{1}{k^{\prime}} \sum k^{i} k^{i}+\frac{1}{k^{\prime \prime}} \sum m^{i} m^{i}-\frac{1}{2} \partial Y \partial Y+T_{F}
$$

as the energy-momentum tensor in spacetime.

Now, we have eight bosons and eight fermions. But this set of variables are not convenient. 
For future convenience, we introduce two new variables to substitute for $\partial \phi$ and $\partial Y$ Define

$$
J^{0}=A \partial \phi+B \partial Y, \quad K^{0}=C \partial \phi+D \partial Y
$$

and

$$
J^{0}(z) J^{0}(w)=K^{0}(z) K^{0}(w)=-\frac{1}{(z-w)^{2}}, \quad J^{0}(z) K^{0}(w)=0
$$

The coefficients $A, B, C, D$ satisfy

$$
A^{2}+B^{2}=C^{2}+D^{2}=A D-B C=1, \quad A C+B D=0
$$

we can choose the solution

$$
A=D=\sqrt{\gamma}, \quad B=-C=-\sqrt{1-\gamma}
$$

where $\gamma=\frac{k^{\prime \prime}}{k^{\prime}+k^{\prime \prime}}$. Of course both $J^{0}$ and $K^{0}$ belong to $(\mathbf{0}, \mathbf{0})$. So the energy-momentum tensor in spacetime can be rewritten as

$$
T(z)=-\frac{1}{2}\left(J^{0}\right)^{2}-\frac{1}{2}\left(K^{0}\right)^{2}-\left(\frac{1}{\alpha_{+}}-\frac{\alpha_{+}}{2}\right) \sqrt{\gamma} \partial J^{0}-\left(\frac{1}{\alpha_{+}}-\frac{\alpha_{+}}{2}\right) \sqrt{1-\gamma} \partial K^{0}+\frac{1}{k^{\prime}} \sum k^{i} k^{i}+\frac{1}{k^{\prime \prime}} \sum m^{i} m^{i}+T_{F}
$$

Now we are ready to construct the large $N=4$ algebra, which consists of sixteen holomorphic currents [22, 20]. Apart from the energy-momentum tensor $T(z)$ and its four superpartners $G_{\alpha \beta}$, there are seven currents $A^{i}, B^{i}, U$, which generate $s u(2) \times s u(2) \times u(1)$ subalgebra, (it arises from the $S^{3} \times S^{3} \times S^{1}$ background, and is the so-called " $R$-symmetry" of the large $N=4$ algebra. The affine $s u(2) \times s u(2) \times u(1)$ algebra in the spacetime $N=4$ SCFT is lifted from the worldsheet.). Finally, there are four weight $\frac{1}{2}$ fermionic generators $Q_{\alpha \beta}$. They satisfy the following OPEs

$$
\begin{aligned}
T(z) T(w)= & \frac{c / 2}{(z-w)^{4}}+\frac{2 T(w)}{(z-w)^{2}}+\frac{\partial T(w)}{z-w} \\
G_{\alpha \beta}(z) G_{\alpha^{\prime} \beta^{\prime}}(w)= & \epsilon_{\alpha \alpha^{\prime}} \epsilon_{\beta \beta^{\prime}}\left[\frac{2 c / 3}{(z-w)^{3}}+\frac{2 T(w)}{z-w}\right]+\left(\sigma_{i}\right)_{\alpha}{ }^{\rho} \epsilon_{\rho \alpha^{\prime}} \epsilon_{\beta \beta^{\prime}}\left[\frac{4 \gamma A^{i}}{(z-w)^{2}}+\frac{2 \gamma \partial A^{i}}{z-w}\right](w) \\
& +\left(\sigma_{i}\right)_{\beta^{\prime}}{ }^{\rho} \epsilon_{\rho \beta} \epsilon_{\alpha \alpha^{\prime}}\left[\frac{4(1-\gamma) B^{i}}{(z-w)^{2}}+\frac{2(1-\gamma) \partial B^{i}}{z-w}\right](w) \\
A^{i}(z) G_{\alpha \beta}(w)= & \frac{1}{2}\left(\sigma_{i}\right)_{\alpha}{ }^{\rho}\left[\frac{G_{\rho \beta}}{z-w}-\frac{2(1-\gamma) Q_{\rho \beta}}{(z-w)^{2}}\right](w) \\
B^{i}(z) G_{\alpha \beta}(w)= & \frac{1}{2}\left(\sigma_{i}\right)_{\beta}{ }^{\rho}\left[\frac{G_{\alpha \rho}(w)}{z-w}+\frac{2 \gamma Q_{\alpha \rho}(w)}{(z-w)^{2}}\right] \\
A^{i}(z) A^{j}(w)= & \frac{k^{\prime} / 2 \delta^{i j}}{(z-w)^{2}}+\frac{i \epsilon^{i j k} A^{k}(w)}{z-w} \\
B^{i}(z) B^{j}(w)= & \frac{k^{\prime \prime} / 2 \delta^{i j}}{(z-w)^{2}}+\frac{i \epsilon^{i j k} B^{k}(w)}{z-w} \\
Q_{\alpha \beta}(z) G_{\alpha^{\prime} \beta^{\prime}}(w)= & \frac{\epsilon_{\alpha \alpha^{\prime}} \epsilon_{\beta \beta^{\prime}} U(w)}{z-w}+\frac{1}{z-w}\left[\left(\sigma_{i}\right)_{\alpha}{ }^{\rho} \epsilon_{\rho \alpha^{\prime}} \epsilon_{\beta \beta^{\prime}} A^{i}-\left(\sigma_{i}\right)_{\beta^{\prime}}{ }^{\rho} \epsilon_{\rho \beta} \epsilon_{\alpha \alpha^{\prime}} B^{i}\right](w)
\end{aligned}
$$




$$
\begin{aligned}
A^{i}(z) Q_{\alpha \beta}(w) & =\frac{1}{2}\left(\sigma_{i}\right)_{\alpha}{ }^{\rho} \frac{Q_{\rho \beta}(w)}{z-w} \\
B^{i}(z) Q_{\alpha \beta}(w) & =\frac{1}{2} \frac{\left(\sigma_{i}\right)_{\beta}{ }^{\rho} Q_{\alpha \rho}(w)}{z-w} \\
U(z) G_{\alpha \beta}(w) & =\frac{Q_{\alpha \beta}(w)}{(z-w)^{2}} \\
U(z) U(w) & =-\frac{c}{12 \gamma(1-\gamma)(z-w)^{2}} \\
Q_{\alpha \beta}(z) Q_{\alpha^{\prime} \beta^{\prime}}(w) & =-\frac{c}{12 \gamma(1-\gamma)} \frac{\epsilon_{\alpha \alpha^{\prime}} \epsilon_{\beta \beta^{\prime}}}{z-w} \\
T(z) \Phi(w) & =\frac{d_{\Phi} \Phi(w)}{(z-w)^{2}}+\frac{\partial \Phi(w)}{z-w}
\end{aligned}
$$

where $\sigma_{i}=\left(\sigma^{i}\right)^{*}, \sigma^{i}, i=1,2,3$ is the Pauli matrix,

$$
\sigma^{1}=\left(\begin{array}{cc}
0 & 1 \\
1 & 0
\end{array}\right), \quad \sigma^{2}=\left(\begin{array}{cc}
0 & -i \\
i & 0
\end{array}\right), \quad \sigma^{3}=\left(\begin{array}{cc}
1 & 0 \\
0 & -1
\end{array}\right)
$$

$\Phi(z)=\left\{G_{\alpha \beta}, A^{i}, B^{i}, U, Q_{\alpha \beta}\right\}$ and $d_{\Phi}=\left\{\frac{3}{2}, 1,1,1, \frac{1}{2}\right\}$ accordingly, $c=6 k$.

We begin with the construction of the $s u(2) \times s u(2) \times u(1)$ subalgebra. As in [21, 19], the worldsheet current algebra pertaining to the first $s u(2)$ can be represented as

$$
A^{i}=k^{i}+\frac{1}{2} \psi_{\alpha} \sigma_{\alpha \beta}^{i} \bar{\psi}_{\beta}+\frac{1}{2} \chi_{\alpha} \sigma_{\alpha \beta}^{i} \bar{\chi}_{\beta}
$$

the explicit expression is

$$
\begin{aligned}
A^{+} & =k^{+}+\psi_{1} \bar{\psi}_{2}+\chi_{1} \bar{\chi}_{2} \\
A^{3} & =k^{3}+\frac{1}{2}\left(\psi_{1} \bar{\psi}_{1}-\psi_{2} \bar{\psi}_{2}\right)+\frac{1}{2}\left(\chi_{1} \bar{\chi}_{1}-\chi_{2} \bar{\chi}_{2}\right) \\
A^{-} & =k^{-}+\psi_{2} \bar{\psi}_{1}+\chi_{2} \bar{\chi}_{1}
\end{aligned}
$$

which belong to $(\mathbf{1}, \mathbf{0})$. It is easy to check that

$$
A^{i}(z) A^{j}(w)=\frac{\delta^{i j} k^{\prime} / 2}{(z-w)^{2}}+\frac{i \epsilon^{i j k} A^{k}(w)}{z-w}
$$

The second set of $s u(2)$ currents can be constructed as follows

$$
\begin{aligned}
B^{+} & =m^{+}+\chi_{1} \chi_{2}+\psi_{1} \psi_{2} \\
B^{3} & =m^{3}+\frac{1}{2}\left(\chi_{1} \bar{\chi}_{1}+\chi_{2} \bar{\chi}_{2}\right)+\frac{1}{2}\left(\psi_{1} \bar{\psi}_{1}+\psi_{2} \bar{\psi}_{2}\right) \\
B^{-} & =m^{-}+\bar{\chi}_{2} \bar{\chi}_{1}+\bar{\psi}_{2} \bar{\psi}_{1}
\end{aligned}
$$

and they satisfy

$$
B^{i}(z) B^{j}(w)=\frac{\delta^{i j} k^{\prime \prime} / 2}{(z-w)^{2}}+\frac{i \epsilon^{i j k} B^{k}(w)}{z-w}
$$


which are in $(\mathbf{0}, \mathbf{1})$. The last $u(1)$ current can be constructed in a simple way

$$
U(z)=-\sqrt{\frac{k^{\prime}}{2}} J^{0}+\sqrt{\frac{k^{\prime \prime}}{2}} K^{0}=\sqrt{\frac{k^{\prime}+k^{\prime \prime}}{2}} \partial Y
$$

Here the coefficients are normalized to satisfy the large $N=4$ algebra. The spacetime affine $s u(2) \times s u(2) \times u(1)$ subalgebra was given in ref. [17]

$$
\left\{A_{n}^{a}, B_{n}^{a}, U_{n}\right\}=\oint d z\left\{A^{a}(z), B^{a}(z), U(z)\right\} \gamma^{n}(z)
$$

In the light-cone gauge, they become simply

$$
\left\{A_{n}^{a}, B_{n}^{a}, U_{n}\right\}=\oint d z\left\{A^{a}(z), B^{a}(z), U(z)\right\} e^{n q} z^{p n}
$$

Obviously, $A_{n}^{a}$ 's and $B_{n}^{a}$ 's form two affine $s u(2)$ Lie algebras with level $k^{\prime}\left(k^{\prime \prime}\right)_{s t}=p k^{\prime}\left(p k^{\prime \prime}\right)$. When $q=0, p=1$ is set, those expression will take rather simpler form,

$$
\left\{A_{n}^{a}, B_{n}^{a}, U_{n}\right\}=\oint d z\left\{A^{a}(z), B^{a}(z), U(z)\right\} z^{n}
$$

So $A^{a}(z), B^{a}(z), U(z)$ can be regarded as affine Lie algebras on the spacetime's boundary.

Now only four fermionic generators $Q_{\alpha \beta}$ and four supercurrents $G_{\alpha \beta}$ are to be determined. They are both in $\left(\frac{\mathbf{1}}{\mathbf{2}}, \frac{\mathbf{1}}{\mathbf{2}}\right)$, so we can write them as $2 \times 2$ matrices

$$
Q=\left(\begin{array}{ll}
Q_{11} & Q_{12} \\
Q_{21} & Q_{22}
\end{array}\right), \quad G=\left(\begin{array}{ll}
G_{11} & G_{12} \\
G_{21} & G_{22}
\end{array}\right)
$$

Clearly, $A^{i}$ belonging to $(\mathbf{1}, \mathbf{0})$ acts on the first index of $Q_{\alpha \beta}\left(G_{\alpha \beta}\right)$ only, $B^{i}$ in $(\mathbf{0}, \mathbf{1})$ acts on the second index. $Q_{11}$ is a combination of $\psi_{1}$ and $\chi_{1}, Q_{11}=A \psi_{1}+B \chi_{1}$, (we hope it will not lead to any confusions with (13)). $Q_{\alpha \beta}$ can be determined by the $A^{i}, B^{i}$ action. The coefficients $A$ and $B$ can be determined by using OPE, $Q_{11}(z) Q_{22}(w)=-\frac{c}{12 \gamma(1-\gamma)} \frac{1}{z-w}$. We can choose, $A=\sqrt{\frac{k^{\prime}}{2}}$, $B=-\sqrt{\frac{k^{\prime \prime}}{2}}$ as the solution. So these four fermionic generators $Q_{\alpha \beta}$ can be expressed explicitly

$$
\begin{array}{cc}
Q_{11}=\sqrt{\frac{k^{\prime}}{2}} \psi_{1}-\sqrt{\frac{k^{\prime \prime}}{2}} \chi_{1}, & Q_{12}=\sqrt{\frac{k^{\prime}}{2}} \bar{\psi}_{2}-\sqrt{\frac{k^{\prime \prime}}{2}} \bar{\chi}_{2} \\
Q_{21}=\sqrt{\frac{k^{\prime}}{2}} \psi_{2}-\sqrt{\frac{k^{\prime \prime}}{2}} \chi_{2}, & Q_{22}=-\sqrt{\frac{k^{\prime}}{2}} \bar{\psi}_{1}+\sqrt{\frac{k^{\prime \prime}}{2}} \bar{\chi}_{1}
\end{array}
$$

Now, we construct the supercurrents. Consider all the possibilities for constructing $G_{11}$ up to normalization factors,

$G_{11}=A\left[k^{3} \psi_{1}+B k^{+} \psi_{2}+C \partial \psi_{1}+D \psi_{1} \bar{\psi}_{2} \psi_{2}+E J^{0} \psi_{1}\right]+F\left[m^{3} \chi_{1}+G m^{+} \bar{\chi}_{2}+H \partial \chi_{1}+I \chi_{1} \bar{\chi}_{2} \chi_{2}+J K^{0} \chi_{1}\right]$

where $A, B, \cdots, J$ (we also hope it will not lead to any ambiguities with (13)) are numeric coefficients to be determined. $A^{+}(z) G_{11}(w)$ regular requires $B=1 . B^{+}(z) G_{11}(w)$ regular determines $G=1$. $G_{11}(z) G_{11}(w)$ regular determines $D=1, E=-\sqrt{\frac{k^{\prime}}{2}}$ and $I=-1, J=-\sqrt{\frac{k^{\prime \prime}}{2}}$. The coefficients $A, F, C, H$ can be determined by considering OPE between $G_{11}$ and $A^{-}$as well 
as $G_{11}$ and $B^{-}$. The other three supercurrets $G_{21}, G_{12}, G_{22}$ can be obtained by acting $A^{-}$and $B^{-}$on $G_{11} \ldots$. In this way, we get all supercurrents and we list them as follows

$$
\begin{aligned}
G_{11}= & -\sqrt{\frac{2}{k^{\prime}}}\left[k^{3} \psi_{1}+k^{+} \psi_{2}+\left(1-\frac{c}{6}\right) \partial \psi_{1}+\psi_{1} \bar{\psi}_{2} \psi_{2}-\sqrt{\frac{k^{\prime}}{2}} J^{0} \psi_{1}\right] \\
& -\sqrt{\frac{2}{k^{\prime \prime}}}\left[m^{3} \chi_{1}+m^{+} \bar{\chi}_{2}+\left(1-\frac{c}{6}\right) \partial \chi_{1}-\chi_{1} \bar{\chi}_{2} \chi_{2}-\sqrt{\frac{k^{\prime \prime}}{2}} K^{0} \chi_{1}\right] \\
G_{21}= & -\sqrt{\frac{2}{k^{\prime}}}\left[-k^{3} \psi_{2}+k^{-} \psi_{1}+\left(1-\frac{c}{6}\right) \partial \psi_{2}+\bar{\psi}_{1} \psi_{1} \psi_{2}-\sqrt{\frac{k^{\prime}}{2}} J^{0} \psi_{2}\right] \\
& -\sqrt{\frac{2}{k^{\prime \prime}}}\left[m^{3} \chi_{2}-m^{+} \bar{\chi}_{1}+\left(1-\frac{c}{6}\right) \partial \chi_{2}-\bar{\chi}_{1} \chi_{1} \chi_{2}-\sqrt{\frac{k^{\prime \prime}}{2}} K^{0} \chi_{2}\right] \\
G_{12}= & \sqrt{\frac{2}{k^{\prime}}}\left[-k^{3} \bar{\psi}_{2}+k^{+} \bar{\psi}_{1}-\left(1-\frac{c}{6}\right) \partial \bar{\psi}_{2}+\bar{\psi}_{1} \psi_{1} \bar{\psi}_{2}+\sqrt{\frac{k^{\prime}}{2}} J^{0} \bar{\psi}_{2}\right] \\
& +\sqrt{\frac{2}{k^{\prime \prime}}}\left[m^{3} \bar{\chi}_{2}-m^{-} \chi_{1}-\left(1-\frac{c}{6}\right) \partial \bar{\chi}_{2}-\bar{\chi}_{1} \chi_{1} \bar{\chi}_{2}+\sqrt{\frac{k^{\prime \prime}}{2}} K^{0} \bar{\chi}_{2}\right] \\
G_{22}= & -\sqrt{\frac{2}{k^{\prime}}}\left[k^{3} \bar{\psi}_{1}+k^{-} \bar{\psi}_{2}-\left(1-\frac{c}{6}\right) \partial \bar{\psi}_{1}+\bar{\psi}_{2} \psi_{2} \bar{\psi}_{1}+\sqrt{\frac{k^{\prime}}{2}} J^{0} \bar{\psi}_{1}\right] \\
& -\sqrt{\frac{2}{k^{\prime \prime}}}\left[m^{3} \bar{\chi}_{1}+m^{-} \chi_{2}-\left(1-\frac{c}{6}\right) \partial \bar{\chi}_{1}-\bar{\chi}_{2} \chi_{2} \bar{\chi}_{1}+\sqrt{\frac{k^{\prime \prime}}{2}} K^{0} \bar{\chi}_{1}\right]
\end{aligned}
$$

where $c=6 k=\frac{6 k^{\prime} k^{\prime \prime}}{k^{\prime}+k^{\prime \prime}}$ 17, 20, as desired. Generators (17), (21), (23), (25), (29), (31), form the complete basis of the large $N=4 \mathrm{SCF}$ algebra. It is a straightforward thing to check that they satisfy the OPEs (18)

\section{$3 \quad$ Middle $N=4$ and $N=3$ spacetime SCFT}

We shall construct the so-called middle $N=4$ and $N=3$ superconformal algebra on the spacetime boundary in this section. For the case of middle $N=4$ superconformal algebra, there are sixteen holomorphic currents [22, 23, 20] too, but the Kac-Moody subalgebra this time is $s u(2) \times u(1)^{4}$ (it arises from the $S^{3} \times T^{4}$ background ).

As pointed out in ref. [20], if either of $k^{\prime}$ or $k^{\prime \prime}$, say, $k^{\prime \prime} \rightarrow \infty$ (it amount to set $\gamma \rightarrow 1$ ), then in the OPE

$$
B^{i}(z) B^{j}(w)=\frac{k^{\prime \prime} / 2 \delta^{i j}}{(z-w)^{2}}+\frac{i \epsilon^{i j k} B^{k}}{z-w}
$$

we can simply neglect the last term. It means that one of the $s u(2)$ Kac-Moody algebra $B^{i}(z)$ is broken to $u(1)^{3}$ Kac-Moody $\hat{B}^{i}(z)$. From the spacetime point of view, it corresponds to the $A d S_{3} \times S^{3} \times T^{4}$ background. The criticality requirement reduces to $k=k^{\prime}$ and the center charge equals $6 k$ now. We shall construct this middle $N=4$ algebra from the large one by means of the Inönö -Winger contraction [22].

Make the following definitions

$$
\hat{T}(z)=\lim _{\gamma \rightarrow 1} T(z), \quad \hat{U}^{i}(z)=\lim _{\gamma \rightarrow 1} \sqrt{1-\gamma} B^{i}(z)
$$




$$
\begin{array}{r}
\hat{G}_{\alpha \beta}(z)=\lim _{\gamma \rightarrow 1} G_{\alpha \beta}(z), \quad \hat{Q}_{\alpha \beta}(z)=\lim _{\gamma \rightarrow 1} \sqrt{1-\gamma} Q_{\alpha \beta}(z) \\
\hat{J}^{i}(z)=\lim _{\gamma \rightarrow 1} A^{i}(z), \quad \hat{U}(z)=\lim _{\gamma \rightarrow 1} \sqrt{1-\gamma} U(z)
\end{array}
$$

It seems that some of them are trivial and some of them have vanishing right hand sides. In fact neither of these observation are correct. The reason has been given in detail in ref.[22]. First look at $\hat{U}^{i}(z) \hat{U}^{j}(w)$, we can easily get $\hat{U}^{i}(z) \hat{U}^{j}(w)=\frac{\delta^{i j} k^{\prime} / 2}{(z-w)^{2}}=\frac{\delta^{i j} c / 12}{(z-w)^{2}}$, so we obtain $u(1)^{3}$ algebra $\hat{B}^{i}(z)$ which is broken from $B^{i}(z)$ as follows,

$$
\hat{B}^{-}=\hat{U}^{-}, \quad \hat{B}^{3}=\hat{U}^{3}, \quad \hat{B}^{+}=\hat{U}^{+}
$$

and other generators (energy-momentum tensor $\hat{T}$, supercurrent $\hat{G}_{i j}$ and four spin $\frac{1}{2}$ fermions $\hat{Q}_{i j}$ ) can be gotten in a similar way. We list these generators in the following,

$$
\begin{aligned}
& \hat{T}(z)=-\frac{1}{2}\left(J^{0}\right)^{2}-\frac{1}{2}\left(K^{0}\right)^{2}-\left(\frac{1}{\alpha_{+}}-\frac{\alpha_{+}}{2}\right) \partial J^{0}+\frac{1}{k^{\prime}} \sum k^{i} k^{i}+\frac{6}{c} \hat{U}^{i} \hat{U}^{i}+\hat{T}_{F} \\
& \hat{G}_{11}=-\sqrt{\frac{2}{k^{\prime}}}\left[k^{3} \psi_{1}+k^{+} \psi_{2}+\left(1-\frac{c}{6}\right) \partial \psi_{1}+\psi_{1} \bar{\psi}_{2} \psi_{2}-\sqrt{\frac{k^{\prime}}{2}} J^{0} \psi_{1}\right] \\
& +\left(K^{0} \chi_{1}-\sqrt{\frac{12}{c}} \hat{U}^{3} \chi_{1}-\sqrt{\frac{12}{c}} \hat{U}^{+} \bar{\chi}_{2}\right) \\
& \hat{G}_{21}=-\sqrt{\frac{2}{k^{\prime}}}\left[-k^{3} \psi_{2}+k^{-} \psi_{1}+\left(1-\frac{c}{6}\right) \partial \psi_{2}+\bar{\psi}_{1} \psi_{1} \psi_{2}-\sqrt{\frac{k^{\prime}}{2}} J^{0} \psi_{2}\right] \\
& +\left(K^{0} \chi_{2}-\sqrt{\frac{12}{c}} \hat{U}^{3} \chi_{2}+\sqrt{\frac{12}{c}} \hat{U}^{+} \bar{\chi}_{1}\right) \\
& \hat{G}_{12}=\sqrt{\frac{2}{k^{\prime}}}\left[-k^{3} \bar{\psi}_{2}+k^{+} \bar{\psi}_{1}-\left(1-\frac{c}{6}\right) \partial \bar{\psi}_{2}+\bar{\psi}_{1} \psi_{1} \bar{\psi}_{2}+\sqrt{\frac{k^{\prime}}{2}} J^{0} \bar{\psi}_{2}\right] \\
& +\left(K^{0} \bar{\chi}_{2}+\sqrt{\frac{12}{c}} \hat{U}^{3} \bar{\chi}_{2}-\sqrt{\frac{12}{c}} \hat{U}^{-} \chi_{1}\right) \\
& \hat{G}_{22}=-\sqrt{\frac{2}{k^{\prime}}}\left[k^{3} \bar{\psi}_{1}+k^{-} \bar{\psi}_{2}-\left(1-\frac{c}{6}\right) \partial \bar{\psi}_{1}+\bar{\psi}_{2} \psi_{2} \bar{\psi}_{1}+\sqrt{\frac{k^{\prime}}{2}} J^{0} \bar{\psi}_{1}\right] \\
& -\left(K^{0} \bar{\chi}_{1}+\sqrt{\frac{12}{c}} \hat{U}^{3} \bar{\chi}_{1}+\sqrt{\frac{12}{c}} \hat{U}^{-} \chi_{2}\right) \\
& \hat{U}(z)=\sqrt{\frac{c}{12}} K^{0} \\
& \hat{B}^{-}=\hat{U}^{-}, \quad \hat{B}^{3}=\hat{U}^{3}, \quad \hat{B}^{+}=\hat{U}^{+} \\
& \hat{Q}_{11}=-\sqrt{\frac{c}{12}} \chi_{1}, \quad \hat{Q}_{12}=-\sqrt{\frac{c}{12}} \bar{\chi}_{2} \\
& \hat{Q}_{21}=-\sqrt{\frac{c}{12}} \chi_{2}, \quad \hat{Q}_{22}=\sqrt{\frac{c}{12}} \bar{\chi}_{1} \\
& \hat{J}^{+}=k^{+}+\psi_{1} \bar{\psi}_{2}+\chi_{1} \bar{\chi}_{2} \\
& \hat{J}^{3}=k^{3}+\frac{1}{2}\left(\psi_{1} \bar{\psi}_{1}-\psi_{2} \bar{\psi}_{2}\right)+\frac{1}{2}\left(\chi_{1} \bar{\chi}_{1}-\chi_{2} \bar{\chi}_{2}\right) \\
& \hat{J}^{-}=k^{-}+\psi_{2} \bar{\psi}_{1}+\chi_{2} \bar{\chi}_{1}
\end{aligned}
$$


where

$$
\hat{T}_{F}=T_{F}=\frac{1}{2}\left(\partial \psi_{1} \bar{\psi}_{1}-\psi_{1} \partial \bar{\psi}_{1}+\partial \psi_{2} \bar{\psi}_{2}-\psi_{2} \partial \bar{\psi}_{2}+\partial \chi_{1} \bar{\chi}_{1}-\chi_{1} \partial \bar{\chi}_{1}+\partial \chi_{2} \bar{\chi}_{2}-\chi_{2} \partial \bar{\chi}_{2}\right)
$$

are unchanged.

They satisfy the following middle $N=4$ superconformal algebra

$$
\begin{aligned}
& \hat{T}(z) \hat{T}(w)=\frac{c / 2}{(z-w)^{4}}+\frac{2 \hat{T}(w)}{(z-w)^{2}}+\frac{\partial \hat{T}(w)}{z-w} \\
& \hat{G}_{\alpha \beta}(z) \hat{G}_{\alpha^{\prime} \beta^{\prime}}(w)=\epsilon_{\alpha \alpha^{\prime}} \epsilon_{\beta \beta^{\prime}}\left[\frac{2 c / 3}{(z-w)^{3}}+\frac{2 \hat{T}(w)}{z-w}\right]+\left(\sigma_{i}\right)_{\alpha}^{\rho} \epsilon_{\rho \alpha^{\prime}} \epsilon_{\beta \beta^{\prime}}\left[\frac{4 \hat{J}^{i}}{(z-w)^{2}}+\frac{2 \partial \hat{J}^{i}}{z-w}\right](w) \\
& \hat{J}^{i}(z) \hat{G}_{\alpha \beta}(w)=\frac{1}{2}\left(\sigma_{i}\right)_{\alpha}{ }^{\rho} \frac{\hat{G}_{\rho \beta}(w)}{z-w} \\
& \hat{B}^{i}(z) G_{\alpha \beta}(w)=\frac{\left(\sigma_{i}\right)_{\beta}{ }^{\rho} \hat{Q}_{\alpha \rho}(w)}{(z-w)^{2}} \\
& \hat{U}(z) \hat{G}_{\alpha \beta}=\frac{\hat{Q}_{\alpha \beta}(w)}{(z-w)^{2}} \\
& \hat{J}^{i}(z) \hat{J}^{j}(w)=\frac{k^{\prime} / 2 \delta^{i j}}{(z-w)^{2}}+\frac{i \epsilon^{i j k} \hat{J}^{k}(w)}{z-w} \\
& \hat{B}^{i}(z) \hat{B}^{j}(w)=\frac{c \delta^{i j}}{12(z-w)^{2}} \\
& \hat{Q}_{\alpha \beta}(z) \hat{G}_{\alpha^{\prime} \beta^{\prime}}(w)=\frac{\epsilon_{\alpha \alpha^{\prime}} \epsilon_{\beta \beta^{\prime}} \hat{U}(w)}{z-w}-\frac{\left(\sigma_{i}\right)_{\beta^{\prime}}{ }^{\rho} \epsilon_{\rho \beta} \epsilon_{\alpha \alpha^{\prime}} \hat{B}^{i}(w)}{z-w} \\
& \hat{Q}_{\alpha \beta}(z) \hat{Q}_{\alpha^{\prime} \beta^{\prime}}(w)=-\frac{c / 12 \epsilon_{\alpha \alpha^{\prime}} \epsilon_{\beta \beta^{\prime}}}{z-w} \\
& \hat{J}^{i}(z) \hat{Q}_{\alpha \beta}(w)=\frac{1}{2}\left(\bar{\sigma}_{i}\right)_{\alpha}{ }^{\rho} \frac{\hat{Q}_{\rho \beta}(w)}{z-w} \\
& \hat{U}(z) \hat{U}(w)=-\frac{c / 12}{(z-w)^{2}} \\
& \hat{U}^{i}(z) \hat{U}(w)^{j}=\frac{c / 12 \delta^{i j}}{(z-w)^{2}} \\
& \hat{T}(z) \hat{\Phi}(w)=\frac{d_{\hat{\Phi}} \hat{\Phi}(w)}{(z-w)^{2}}+\frac{\partial \hat{\Phi}(w)}{z-w}
\end{aligned}
$$

where $\hat{\Phi}=\left\{\hat{G}_{\alpha \beta}, \hat{B}^{i}, \hat{U}, \hat{J}^{i}, \hat{Q}_{\alpha \beta}\right\}$, accordingly, $d_{\hat{\Phi}}$ equal to $\left\{\frac{3}{2}, 1,1,1, \frac{1}{2}\right\}$, and $c=6 k$, as mentioned above.

Recently, string theory on the $A d S_{3} \times\left(S^{3} \times S^{3} \times S^{1}\right) / Z_{2}$ was investigated in ref. 24, and the spacetime $N=3$ superconformal theories was constructed. We shall construct the same algebra in terms of the transversal physical degrees of freedom.

We set $k^{\prime}=k^{\prime \prime}$ in our large $N=4$ algebra. Once this setting is performed, there is an automorphism in our large $N=4$ algebra, namely, a $Z_{2}$ action 24,

$$
\left(A^{i}(z), B^{i}(z), Y(z)\right) \rightarrow\left(B^{i}(z), A^{i}(z),-Y(z)\right)
$$


We consider the eigenstates of the $Z_{2}$ action. Those with eigenvalue +1 are invariant under $Z_{2}$ and will form a subalgebra of the large $N=4$ algebra. It can be checked that the diagonal part of the $s u(2) \times s u(2)$ algebra is invariant under $Z_{2}$ and the isometry $s u(2) \times s u(2)$ of the large $N=4$ is reduced to the diagonal $s u(2)$.

The diagonal $s u(2)$ is invariant under $Z_{2}$, so we can construct our affine Lie algebra uniquely by summing those two $s u(2) \mathrm{s}$

$$
J^{i}(z)=A^{i}(z)+B^{i}(z)
$$

Supercharges $G_{i j}$ belong to the $\left(\frac{\mathbf{1}}{\mathbf{2}}, \frac{\mathbf{1}}{\mathbf{2}}\right)$ representation of $s u(2) \times s u(2)$, and they are decomposed in to $\mathbf{1} \oplus \mathbf{0}$ under the diagonal $s u(2)$. Now we shall explain that only the triplet is $Z_{2}$ invariant. Under the $Z_{2}$ action, two su(2)s are interchanged and at the same time $Y$ is reflected. In our free field realization, this correspond to $A^{i}$ and $B^{i}$ interchanged, and $U$ reflected simultaneously according to (25). Supercurrents and four fermions transform as

$$
Q=\left(\begin{array}{ll}
Q_{11} & Q_{12} \\
Q_{21} & Q_{22}
\end{array}\right) \rightarrow-\left(\begin{array}{ll}
Q_{11} & Q_{21} \\
Q_{12} & Q_{22}
\end{array}\right), G=\left(\begin{array}{ll}
G_{11} & G_{12} \\
G_{21} & G_{22}
\end{array}\right) \rightarrow\left(\begin{array}{ll}
G_{11} & G_{21} \\
G_{12} & G_{22}
\end{array}\right)
$$

It is not very difficult to check that after $Z_{2}$ action, those set of generators still satisfy OPEs (18), except $A^{i}, B^{i}$ interchanged, and the index of supercurrents also exchanged. So only triplet is invariant under the $Z_{2}$ action. Closure of this algebra need a spin $\frac{1}{2}$ fermionic current $\Psi$. We list our $N=3$ algebra generators in the following

$$
\begin{aligned}
T(z)= & -\frac{1}{2}\left(J^{0}\right)^{2}-\frac{1}{2}\left(K^{0}\right)^{2}-\frac{\sqrt{2}}{2}\left(\frac{1}{\alpha_{+}}-\frac{\alpha_{+}}{2}\right) \partial J^{0}-\frac{\sqrt{2}}{2}\left(\frac{1}{\alpha_{+}}-\frac{\alpha_{+}}{2}\right) \partial K^{0} \\
& +\frac{1}{k^{\prime}} \sum k^{i} k^{i}+\frac{1}{k^{\prime \prime}} \sum m^{i} m^{i}+T_{F} \\
T_{F}= & \frac{1}{2}\left(\partial \psi_{1} \bar{\psi}_{1}-\psi_{1} \partial \bar{\psi}_{1}+\partial \psi_{2} \bar{\psi}_{2}-\psi_{2} \partial \bar{\psi}_{2}+\partial \chi_{1} \bar{\chi}_{1}-\chi_{1} \partial \bar{\chi}_{1}+\partial \chi_{2} \bar{\chi}_{2}-\chi_{2} \partial \bar{\chi}_{2}\right) \\
J^{+}= & A^{+}+B^{+}, \quad J^{3}=A^{3}+B^{3}, \quad J^{-}=A^{-}+B^{-} \\
G^{+}= & i \sqrt{2} G_{11}, \quad G^{3}=-i \frac{\sqrt{2}}{2}\left(G_{12}+G_{21}\right), \quad G^{-}=-i \sqrt{2} G_{22} \\
\Psi= & -i \frac{\sqrt{2}}{2}\left(Q_{21}-Q_{12}\right)
\end{aligned}
$$

where the coefficients are normalized to satisfy the following OPEs

$$
\begin{aligned}
T(z) T(w) & =\frac{c / 2}{(z-w)^{4}}+\frac{2 T(w)}{(z-w)^{2}}+\frac{\partial T(w)}{z-w} \\
J^{i}(z) J^{j}(w) & =\frac{k^{\prime} \delta^{i j}}{(z-w)^{2}}+\frac{i \epsilon^{i j k} J^{k}(w)}{z-w} \\
J^{i}(z) G^{j}(w) & =\frac{i \epsilon^{i j k} G^{k}(w)}{z-w}+\frac{\delta^{i j} \Psi(w)}{(z-w)^{2}} \\
G^{i}(z) G^{j}(w) & =\frac{2 c / 3 \delta^{i j}}{(z-w)^{3}}+\frac{2 i \epsilon^{i j k} J^{k}(w)}{(z-w)^{2}}+\frac{i \epsilon^{i j k} \partial J^{k}}{z-w}+\frac{2 \delta^{i j} T(w)}{z-w} \\
\Psi(z) \Psi(w) & =\frac{k^{\prime}}{z-w} \\
T(z) \hat{\Phi}(w) & =\frac{d_{\hat{\Phi} \hat{\Phi}(w)}}{(z-w)^{2}}+\frac{\partial \hat{\Phi}(w)}{z-w}
\end{aligned}
$$


where $\hat{\Phi}=\left\{J^{i}, G^{i}, \Psi\right\}, d_{\hat{\Phi}}=\left\{1, \frac{3}{2}, \frac{1}{2}\right\}$, and the center charge now is $c=6 k=3 k^{\prime}=3 k^{\prime \prime}$ (remember $k=\frac{k^{\prime}}{2}=\frac{k^{\prime \prime}}{2}$ in this case).

\section{Conclusions and Remarks}

To have a better understanding of the $A d S / C F T$ correspondence, it is essential to construct the spacetime boundary CFT explicitly. In this note we have realized the spacetime SCF algebras explicitly in terms of transversal degrees of freedom, corresponding to strings propagating on $A d S_{3} \times S^{3} \times S^{3} \times S^{1}, A d S_{3} \times S^{3} \times T^{4}$ and $A d S_{3} \times\left(S^{3} \times S^{3} \times S^{1}\right) / Z_{2}$ respectively. This is a modest step towards to full construction of the spacetime CFT living on the $A d S_{3}$ boundary. As in the case of the large $N=4 \mathrm{SCF}$ algebra [20], we may consider the spacetime SCFT as some twisted version of $p$ copies of nonlinear sigma model, each with center charge $6 k$. It remains to check the correspondence between the string theory in the bulk and the CFT on the boundary.

A manifest similarity between the two theory is that the DDF state [25, 26] on the string theory side resembles very much to the conformal algebra on the boundary CFT side, except that the energy momentum tensor on the each side differ by a improved term. Does that means some duality between the two CFT? It deserves further clarification.

\section{Acknowledgment}

One of the authors (J. Jing) would like to appreciate Dr. Bihn Zhou and Dr. Shi-hua Zhao for their constructive discussions and enthusiastic helps.

\section{References}

[1] J.Balog, L.O'Raifeartaigh, P.Forgacs and Wipf, Nucl.Phys B325, (1989) 225

[2] I.Bars, Ghost-Free Spectrum of a Quantum String in SL(2,R) Curved Spacetime, hepth/9503205(1995), Phys.Rev. D53 (1996) 3308-3323

[3] I.Bars, Solution of the SL(2,R) string in curved spacetime, hep-th/9511187

[4] Y.Satoh, Ghost-free and Modular Invariant Spectra of a String in SL(2,R) and Three Dimensional Black Hole Geometry, hep-th/9705208(1997), Nucl.Phys. B513 (1998) 213228 ,

[5] J. Maldacena, The Large N Limit of Superconformal Field Theories and Supergravity, hepth/9711200 (1997), Adv. Theor. Math. Phys.2(1998)231

[6] I.Bars, String Theory on AdS(3) Revisited, hep-th/9907087

[7] K. Hosomichi, Y. Sugawara Hilbert Space of Space-time SCFT in AdS S Supersting and $T^{4 k p} / S_{k p} S C F T$, hep-th/9812100 (1998), JHEP 9901 (1999) 013

[8] K. Hosomichi, Y. Sugawara Multi-Strings on $A d S_{3} \times S^{3}$ from Matrix String Theory, hepth/9905004 (1999), JHEP 9907 (1999) 027 
[9] Y. Sugawara, Topological String on $A d S_{3} \times N$, hep-th/9909146 (1999), Nucl.Phys. B576 (2000) 265-284

[10] P.M.Petropoulos String Theory on AdS $S_{3}$ Some Open Questions, hep-th/9908189

[11] Oleg.Andreev, On Affine Lie Superalgebras, AdS $S_{3} / C F T$ Correspondence And WorldSheets For World-Sheets, hep-th/9901118 (1999), Nucl.Phys. B552 (1999) 169-193,

Oleg.Andreev, Unitary Representations of Some Infinite Dimensional Lie Algebras Motivated by String Theory on AdS $S_{3}$, hep-th/9905002 (1999), Nucl.Phys. B561 (1999) 413-432,

[12] Jan de Boer, Hirosi Ooguri, Harlan Robins, Jonathan Tannenhauser, String Theory on $A d S_{3}$, hep-th/9812046 (1998), JHEP 9812 (1998) 026

Jan de Boer, Andrea Pasquinucci, Kostas Skenderis, AdS/CFT dualities involving large 2d $N=4$ superconformal symmetry, hep-th/9904073 (1999),

Jan de Boer, Samson L. Shatashvili, Two-dimensional Conformal Field Theories on $A d S_{2 d+1}$ Backgrounds, hep-th/9905032 (1999), JHEP 9906 (1999) 013,

[13] O.Aharony, S.S.Gubser, J.Maldacena, H.Ooguri, Y.Oz, Large N Field Theories, String Theory and Gravity, hep-th/9905111 (1999), Phys.Rept. 323(2000)183-386

[14] N.Seiberg, E.Witten, The D1/D5 System and Singular CFT, hep-th/9903224 (1999), JHEP 9904(1999)017

[15] E.Witten, Anti-de Sitter Space and Holography, hep-th/9802150(1998), Adv. Theor. Math. Phys.2(1998), 253.

[16] S.S. Gubser, I.R. Klebanov and A.M. Polyakov, Gauge Theory Correlators from NonCritical String Theory, hep-th/9802109 (1998), Phys.Lett. B428 (1998), 105.

[17] Amit Giveon, David Kutasov and Nathan Seiberg, Comments on String Theory on AdS $S_{3}$, hep-th/9806194 (1998).

[18] D. Kutasov and N. Seiberg More comments on string theory on $A d S_{3}$, hep-th/9903219, JHEP 9904 (1999) 008.

[19] M.Yu, The Unitary Constructions of the N=4 SU(2) Extended Superconformal Algebra, Phys. Lett. B196(1987) 345.

M.Yu, The Unitary Representations of the $N=4 S U(2)$ Extended Superconformal Algebras, Nucl. Phys. B294(1987) 890.

[20] S. Elitzur, O. Feinerman, A. Giveon and D. Tsabar, String Theory on $A d S_{3} \times S^{3} \times S^{3} \times S_{1}$, hep-th/9811245(1998).

[21] M. Yu, B. Zhang, Light-Cone Gauge Quantization of String Theories on AdS 3 Space, hep-th/9812216, Nucl.Phys. B551 (1999) 425-449

[22] Abbas Ali, Classification of Two Dimensional N=4 Superconformal Symmetries, hepth/9906096

[23] Abbas Ali, Alok Kumar, A New N=4 Superconformal Algebra, hep-th/9301010 
[24] S. Yamaguchi, Y. Ishimoto, K. Sugiyama, $A d S_{3} / C F T_{2}$ Correspondence and Space-Time $N=3$ Superconformal Algebra, hep-th/9902079 JHEP 9902 (1999) 026

[25] M. Green, J. Schwarz and E. Witten, Superstring Theory, Vol. I, II , Cambridge Univ. Press (1987)

[26] Y Hikida, K Hosomichi, Y Sugawara, String Theory on $A d S_{3}$ as Discrete Light-Cone Liouville Theory, hep-th/0005065 